\title{
Influences of retinal axons on the cultural substrate containing biotin-conjugated
}

chondroitin sulfate in vitro

Satoru Ando ${ }^{1}$, Nobuo Sugiura ${ }^{2}$, Koji Kimata ${ }^{2}$, Hiroyuki Ichijo ${ }^{1}$

${ }^{1}$ Department of Anatomy and Embryology, Institute of Basic Medical Sciences, Graduate School of Comprehensive Human Sciences, University of Tsukuba, Tsukuba, Ibaraki 305-8575, Japan. ${ }^{2}$ Institute for Molecular Science of Medicine and Research Complex for the Medicine Frontiers, Aichi Medical University, Yazako, Nagakute, Aichi 480-1195, Japan.

Short title: Chondroitin sulfate and retinal axons

Number of text pages: 12

Number of figures: 2

Corresponding author: Hiroyuki Ichijo

Department of Anatomy and Embryology, Institute of Basic Medical Sciences, Graduate School of Comprehensive Human Sciences, University of Tsukuba, Tsukuba, Ibaraki 305-8575, Japan.

E-mail: ichijo@md.tsukuba.ac.jp

Phone: +81-29-853-6963

Fax: +81-29-853-6965 


\begin{abstract}
Although chondroitin sulfate (CS) is known to act as an inhibitory axon guidance cue, retinal axons show substantial growth on a culture substrate containing CS. Thus, the question arises as to how retinal axons elongate on CS-containing culture substrates. To elucidate the effects of retinal axons on a substrate containing CS, we synthesized biotinylated CS (biotin-CS) and developed a culture substrate with streptavidin-conjugated biotin-CS (complex between streptavidin and biotin-CS) to culture retinal axons. The effects of retinal axons on the streptavidin-biotin-CS complex were analyzed immunocytochemically using antibodies against CS and streptavidin, which recognize the carbohydrate and protein portions of the complex, respectively. After the axons were cultured on the substrate, areas that were CS-immunonegative but streptavidin-immunopositive were observed on the surface, corresponding to areas with or without axons, respectively. Absence of CS immunostaining was considered to be caused by structural alterations in the carbohydrate chains of the CS under the influence of the axons.
\end{abstract}

Keywords: Retina, Axon, Chondroitin sulfate, Optic tract 


\section{Introduction}

Neuronal circuit formation is one of the basic themes in neuroscience. Retinotectal projections are good models for studying axon guidance. A series of guidance cues helps axons navigate along an appropriate path. Chondroitin sulfate proteoglycans (CSPGs) are known to act as guidance cues or modulators that exert inhibitory effects on axonal growth at various decision points along the retinotectal pathway. In the retina, CSPGs play roles in regulating the direction of axonal growth of retinal ganglion cells (RGCs) toward the central retina (Brittis et al. 1992). CSPGs inhibit axonal growth of RGCs and contribute to retinotectal pathway formation (Ichijo and Kawabata 2001; Walz et al. 2002; Becker and Becker 2002). CSPGs are involved in the formation of the dorsal midline barrier in the optic tectum (Wu et al. 1995). In the mouse optic chiasm, whether RGCs project their axons ipsilaterally or contralaterally is influenced by chondroitin sulfate (CS) (Chung et al. 2000). In the optic tract, early- and late-growing RGC axons run in the deep and superficial peripheral layers, respectively; thus, RGC axons are ordered chronologically. Removal of CS disrupts this chronological order in the tract (Leung et al. 2003).

CSPGs consist of a core protein and carbohydrate chain of CS. CS is a long unbranched polymer consisting of repeating disaccharide units of glucuronic acid and $\mathrm{N}$-acetylgalactosamine. The units show structural diversity, and the different types are designated A-, B-, C-, D-, and E-unit. Some monoclonal antibodies recognize CSs. One of them-MO-225—preferentially recognizes D-unit- and E-unit-rich CSs (Yamagata et al. 1987; Deepa et al. 2007). The neuropil beneath the optic tract was labeled intensely with MO-225, suggesting that the MO-225-immunopositive neuropil delimits the boundary of the axonal pathway (Ichijo 2006). However, retinal axons run in the deep layer of the optic tract in contact with the MO-225-immunopositive neuropil, which is thought to inhibit axonal growth. Moreover, retinal axons show substantial but not profuse growth on substrates with MO-225-immunopositive CS in vitro. Thus, the question arises as to how axons elongate on a substrate containing MO-225-immunopositive CS. 
To examine whether retinal axons exert an influence on their microenvironment to promote their own growth, we synthesized biotinylated CS (biotin-CS). In particular, D-unit-rich CS (CSD) was biotinylated (biotin-CSD) because it is recognized preferentially by MO-225. We developed a streptavidin/biotin-CSD complex that mimics CSPG and made retinal axons grow on a substrate made of this complex. The results indicated that the carbohydrate portion (CS) of the complex was altered under the influence of the axons. This alteration of CS might be an adaptation of the axons to improve the microenvironment for their growth.

\section{Methods}

All experimental protocols were approved by the Committee for Animal Care and Use of the University of Tsukuba. The reducing ends of CSD (Seikagaku, Tokyo, Japan) were coupled chemically to biotin (Shinohara et al. 1995) to synthesize biotin-CSD. Biotin-CSD had a conjugation efficiency of $21 \%$ and an average molecular weight of $20 \mathrm{kDa}$. Biotin-CSD $(10 \mu \mathrm{l}$ of $0.75 \mu \mathrm{g} / \mu \mathrm{l})$ was incubated with streptavidin $(10 \mu \mathrm{l}$ of $0.075 \mu \mathrm{g} / \mu \mathrm{l})$ for $3 \mathrm{~h}$ at $37.5^{\circ} \mathrm{C}$ to form a complex consisting of a protein core and a carbohydrate chain. An excess of biotin-CSD was used for complex formation because unconjugated CSD was also included in the preparation. The mixtures (10 $\mu$ l per lane) were electrophoresed on $10 \%$ non-denaturing polyacrylamide gels using a running buffer without sodium dodecyl sulfate. After washing twice (in 25\% ethanol, $7.5 \%$ acetic acid, and $0.1 \%$ sodium dodecyl sulfate) for 20 min, the gels were stained with SYPRO Ruby (Bio-Rad, Hercules, CA) and observed with a Fluoroimager 595 (GE Healthcare Bio-Science, Piscataway, NJ). Subsequently, the gels were stained with 0.125\% Alcian blue (Sigma-Aldrich, St. Louis, MO). The Alcian blue-stained gels were photographed using an image scanner (Canon, Tokyo, Japan).

Glass coverslips were coated with poly-D-lysine $(10 \mu \mathrm{g} / \mathrm{ml})$ for $2 \mathrm{~h}$ at $37.5^{\circ} \mathrm{C}$, washed, and air-dried. The coverslips were coated with nitrocellulose dissolved in methanol and again coated with poly-D-lysine (Lagenaur and Lemmon 1987; Becker and Becker 2002). 
Each coverslip was coated with $10 \mu \mathrm{l}$ of the biotin-CSD $(0.1 \mu \mathrm{g} / \mu \mathrm{l})$ and streptavidin $(0.01$ $\mu \mathrm{g} / \mu \mathrm{l})$ mixture and placed in a humid chamber overnight at $37.5^{\circ} \mathrm{C}$. The coverslips were washed in phosphate-buffered saline (PBS) and subsequently coated with laminin (40 $\mu \mathrm{g} / \mathrm{ml}$, Invitrogen, Carlsbad, CA) for $3 \mathrm{~h}$ at $37.5^{\circ} \mathrm{C}$. To confirm formation of the substrate, the coverslips were immunocytochemically double-stained with anti-CS monoclonal antibody MO-225 (Seikagaku) and anti-streptavidin polyclonal antibody (Abcam, Cambridge, UK) along with appropriate secondary antibodies (Invitrogen). The substrate was then used for retinal explant culture.

Retinal explants were prepared from embryonic day 6 chicks, as described previously (Ichijo and Bonhoeffer 1998). They were cultured on the substrate containing the streptavidin-biotin-CSD complex with F12 nutrient mixture supplemented with $8 \%$ fetal bovine serum, $2 \%$ chick serum, and $2 \mathrm{mM} \mathrm{L-glutamine} \mathrm{(Invitrogen)} \mathrm{at} 37.5^{\circ} \mathrm{C}$ in $4 \% \mathrm{CO}_{2}$ for 2 days. The cultures were fixed with 3.7\% formaldehyde in PBS at room temperature for $2 \mathrm{~h}$. In some cultures, the retinal axons were removed, either with forceps or by freeze-thawing, before fixation. The cultures were washed with 0.5\% Triton X-100 in PBS.

To visualize the axons with microfilaments and microtubules under red fluorescence, we used a cocktail of Alexa594-phalloidin (Invitrogen) and anti- $\beta$-tubulin rabbit-polyclonal antibody (Lab Vision, Westinghouse, CA) with anti-rabbit IgG-conjugated Alexa594 (Invitrogen). The cultures were double-stained using the cocktail and MO-225 with anti-mouse IgG-conjugated Alexa488 (Invitrogen). Some cultures were double-stained using MO-225 and anti-streptavidin polyclonal antibody along with appropriate secondary antibodies. All specimens were observed under a fluorescence microscope (DM-XRA2, Leica, Wetzlar, Germany). Digital images were processed with Adobe Photoshop CS, by adjusting for contrast.

\section{Results}

Formation of streptavidin and biotin-CSD complex and development of a culture 


\section{substrate using this complex}

Biotin-CSD incubated with streptavidin was electrophoresed on non-denaturing gels (Fig. 1a).

A broad band stained with both SYPRO Ruby and Alcian blue was observed, indicating formation of a complex between streptavidin and biotin-CSD. Unlike the sharp band for streptavidin, the band for the complex was broad and in the higher molecular-weight range, which is thought to be because of the variation in the molecular weights and anionic charges of the complex with biotin-CSD of varying lengths (average molecular weight, $20 \mathrm{kDa}$ ). Apart from the band corresponding to the complex, a smeared band was observed with Alcian Blue staining, but not with SYPRO Ruby staining. This band represented unconjugated CSD because the efficiency of CS-biotinylation was only $21 \%$.

The substrate for axonal culture was prepared using the streptavidin-biotin-CSD complex. This substrate was double-stained with MO-225 and anti-streptavidin (Fig. 1b, c). Both the antibodies showed uniform staining throughout the substrate, indicating an even distribution of the complex in the substrate, but no antibody staining was observed outside the substrate.

\section{Influence of retinal axons on culture substrate containing the streptavidin-biotin-CSD complex}

Distribution of the streptavidin-biotin-CSD complex on the substrate was assessed by staining with MO-225 and comparing with axonal paths (Fig. 2a-c). In the cultures, some axons were labeled by MO-225 (Fig. 2a). There were two populations of retinal axons with or without MO-225 staining in vitro as shown previously (Ichijo 2006).

There were areas on the substrate where MO-225 staining was absent along some but not all axons (Fig. 2a-c). Areas where MO-225 staining was absent with no axons were also seen. The MO-225-negative areas were aligned like axonal paths. The MO-225-negative areas were observed only on the substrate with cultured axons.

Distribution of the complex was further examined by double staining the substrate with MO-225 and anti-streptavidin after mechanical removal of axons (Fig. 2d-f). Aligned areas on the substrate where MO-225 staining was absent were seen. However, the substrate 
was uniformly stained with anti-streptavidin. The uniform staining of anti-streptavidin indicated that the complex was distributed uniformly on the substrate, and that antibodies readily accessed the complex within the substrate from which axons were mechanically removed. In the MO-225-negative areas, staining was absent in the carbohydrate portion of the complex, CS, but was present in the protein portion, streptavidin.

\section{Discussion}

\section{Technical advantages of using the streptavidin-biotin-CSD complex in vitro}

Streptavidin and biotin-CSD efficiently formed a complex, which is thought to comprise a tetramer with a subunit of one streptavidin binding to one biotin-CSD (Green 1990). This complex offers some advantages. The CS in the complex is indirectly fixed because streptavidin is fixable with formaldehyde, which made it possible to examine detailed distribution of the complex. The absence of MO-225 staining in some regions of the substrate was not due to the uneven distribution of the complex because this was observed only with the axons cultured on the complex. Moreover, the uniform anti-streptavidin staining on the substrate confirmed that the absence of MO-225 staining was not caused by the inability of the antibody to access the complex. The double immunostaining using the antibodies against CS and streptavidin indicated that the complex was under the influence of the growing axons in vitro. This method enabled us to examine whether the axons exert their influence on the microenvironment for their growth. In addition, the complex will be useful to investigate the relationship between the structural diversity of CS and its functional specificity because the combination of each biotin-CS and streptavidin will produce a complex with CS of a different structure.

\section{The influence of axons on the streptavidin-biotin-CSD complex in the substrate in vitro}

Some areas on the substrate were stained with anti-streptavidin but not with MO-225. The MO-225 staining was absent along some, but not all, axons on the substrate (Fig. 2a-c). After mechanical removal of the axons, the areas without MO-225 staining were distinct (Fig. 
2d). This might be because some of the axons labeled with MO-225 (Fig. 2a) might have masked the areas without MO-225 staining in the substrate. Further, some areas without MO-225 staining did not contain axons, but were aligned like axon paths (Fig. 2a). This “axon-less” path was likely to be caused by the tracks of the axons that had grown and then retracted. However, it is unlikely that these could have formed due to the deliberate removal of axons during the staining procedure because such areas usually have remnants of the axons (Fig. 2d-f).

The results indicated that the carbohydrate portion of the complex in the substrate, CS, was altered under the influence of the axons. MO-225 immunostaining was absent in the carbohydrate portion of the complex. The absence of immunostaining is thought to be caused by structural alteration of the CS chains. The results suggest two possibilities. (1) CS chains might be cleaved and removed, for example, by enzymes like hyaluronidases or chondroitinases (Stern and Jedrzejas 2006); or (2) the epitopes of MO-225 might be modified due to the addition or removal of sulfate groups on the CS chains (Morimoto-Tomita et al. 2002; Nagamine et al. 2005).

The protein portion of the substrate complex in the substraet, streptavidin, was not altered (Fig. 2e) although axons are known to release proteolytic enzymes during their growth (Krystosek and Seeds 1981; Seeds et al. 1997; Webber et al. 2002; Pizzi and Crowe 2007). This might be due to the specificity of antibody detection, i.e., polypeptides bearing epitopes recognized by the polyclonal anti-streptavidin antibody remained on the substrate even after their partial degradation.

\section{Functional significance of the alteration of biotin-CSD under the influence of retinal axons}

It has been suggested that the neuropil, with MO-225-immunopositive CS, delimits the boundary of the axonal pathway beneath the optic tract due to the inhibitory effects of CS on axonal growth (Ichijo 2006). However, retinal axons run in contact with the MO-225-immunopositive neuropil in the deep layer of the optic tract in vivo. Here, we showed that in vitro some retinal axons grow on substrates by altering MO-225-immunopositive 
biotin-CSD. This alteration might be an adaptation of the retinal axons to improve the microenvironment for their growth; this might also be the case in vivo when retinal axons grow in contact with the MO-225-immunopositive neuropil in the deep layer of the optic tract.

\section{Acknowledgments}

This work was supported by Grants-in-Aid for the 21st Century COE Program from the Ministry of Education, Culture, Sports, Science, and Technology, Japan, and for Scientific Research (C) (18500234) from the Japan Society for the Promotion of Science. The authors are grateful to Dr. N. Maeda and Prof. S. Takahashi for discussions.

\section{References}

Becker CG, Becker T (2002) Repellent guidance of regenerating optic axons by chondroitin sulfate glycosaminoglycans in zebrafish. J Neurosci 22:842-853.

Brittis PA, Canning DR, Silver J (1992) Chondroitin sulfate as a regulator of neuronal patterning in the retina. Science 255:733-736.

Chung KY, Taylor JS, Shum DK, Chan SO (2000) Axon routing at the optic chiasm after enzymatic removal of chondroitin sulfate in mouse embryos. Development 127:2673-2683.

Deepa SS, Kalayanamitra K, Ito Y, Kongtawelert P, Fukui S, Yamada S, Mikami T, Sugahara K (2007) Novel sulfated octa- and decasaccharides from squid cartilage chondroitin sulfate E: sequencing and application for determination of the epitope structure of the monoclonal antibody MO-225. Biochemistry 46:2453-2465.

Green NM (1990) Avidin and streptavidin. Methods Enzymol 184:51-67.

Ichijo H (2006) Restricted distribution of D-unit-rich chondroitin sulfate carbohydrate chains in the neuropil encircling the optic tract and on a subset of retinal axons in chick embryos. J Comp Neurol 495:470-479.

Ichijo H, Bonhoeffer F (1998) Differential withdrawal of retinal axons induced by a secreted 
factor. J Neurosci 18:5008-5018.

Ichijo H, Kawabata I (2001) Roles of the telencephalic cells and their chondroitin sulfate proteoglycans in delimiting an anterior border of the retinal pathway. $\mathrm{J}$ Neurosci 21:9304-9314.

Krystosek A, Seeds NW (1981) Plasminogen activator release at the neuronal growth cone. Science 213:1532-1534.

Lagenaur C, Lemmon V (1987) An L1-like molecule, the 8D9 antigen, is a potent substrate for neurite extension. Proc Natl Acad Sci USA 84:7753-7757.

Leung K-M, Taylor JSH, Chan S-O (2003) Enzymatic removal of chondroitin sulphates abolishes the age-related axon order in the optic tract of mouse embryos. Eur J Neurosci $17: 1755-1767$.

Morimoto-Tomita M, Uchimura K, Werb Z, Hemmerich S, Rosen SD (2002) Cloning and characterization of two extracellular heparin-degrading endosulfatases in mice and humans. J Biol Chem 277:49175-49185.

Nagamine S, Koike S, Keino-Masu K, Masu M (2005) Expression of a heparan sulfate remodeling enzyme, heparan sulfate 6-O-endosulfatase sulfatase FP2, in the rat nervous system. Brain Res Dev Brain Res 159:135-143.

Pizzi MA, Crowe MJ (2007) Matrix metalloproteinases and proteoglycans in axonal regeneration. Exp Neurol 204:496-511.

Seeds NW, Siconolfi LB, Haffke SP (1997) Neuronal extracellular proteases facilitate cell migration, axonal growth, and pathfinding. Cell Tissue Res 290:367-370.

Shinohara Y, Sota H, Kim F, et al. (1995) Use of a biosensor based on surface plasmon resonance and biotinyl glycans for analysis of sugar binding specificities of lectins. J Biochem 117:1076-1082.

Stern R, Jedrzejas MJ (2006) Hyaluronidases: their genomics, structures, and mechanisms of action. Chem Rev 106:818-839.

Walz A, Anderson RB, Irie A, Chien C-B, Holt CE (2002) Chondroitin sulfate disrupts axon 
pathfinding in the optic tract and alters growth cone dynamics. J Neurobiol 53:330-342.

Webber CA, Hocking JC, Yong VW, Stange CL, Mcfarlane S (2002) Metalloproteases and guidance of retinal axons in the developing visual system. J Neurosci 22:8091-8100.

Wu DY, Jhaveri S, Schneider GE (1995) Glial environment in the developing superior colliculus of hamsters in relation to the timing of retinal axon ingrowth. J Comp Neurol 358:206-218.

Yamagata M, Kimata Y, Oike Y, Tani K, Maeda N, Yoshida K, Simomura Y, Yoneda M, Suzuki S (1987) A monoclonal antibody that specifically recognizes a glucuronic acid 2-sulfate-containing determinant in intact chondroitin sulfate chain. J Biol Chem 262:4146-4152. 


\section{Figure legends}

Fig. 1

Substrate comprising a complex of streptavidin and biotin-CSD. a Non-denaturing polyacrylamide gel double-stained with SYPRO Ruby (1-3) or Alcian blue (1'-3'). Samples loaded are shown below each lanes: 1,1' streptavidin; 2,2' biotin-CSD; 3,3' mixed solution with streptavidin and biotin-CSD. Open arrowheads Complex of streptavidin and biotin-CSD, closed arrowhead streptavidin, asterisk unconjugated CSD. b, c Substrate of the streptavidin-biotin-CSD complex is shown. The substrate is double-stained with MO-225 (b) and anti-streptavidin (c). Broken lines Boundary of the coating. Bars $20 \mu \mathrm{m}$.

Fig. 2

Streptavidin-biotin-CSD complex under the influence of retinal axons in vitro. a-c Retinal culture on streptavidin-biotin-CSD complex substrate, Staining: a MO-225, b axons, c merged image. Arrows indicate absence of MO-225 staining under the axons, arrowheads indicate absence of MO-225 staining with no axons; the MO-225-negative areas are aligned like axon

paths. d-f After mechanical removal of axons. Staining: d MO-225 staining, e anti-streptavidin staining, f merged image. Open arrowheads indicate the absence of MO-225 staining and presence of anti-streptavidin staining along the axon paths. Bars $20 \mu \mathrm{m}$. 


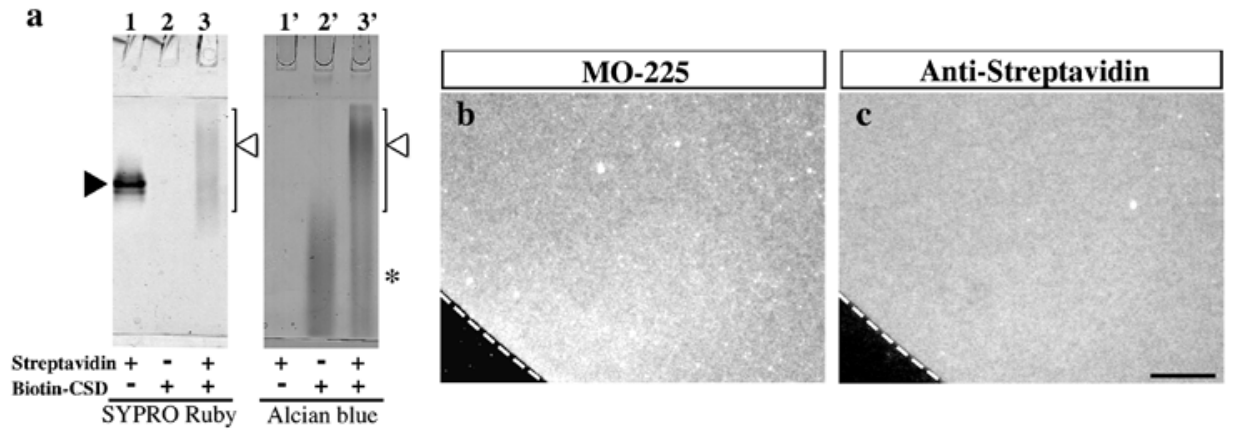

Figure 1 

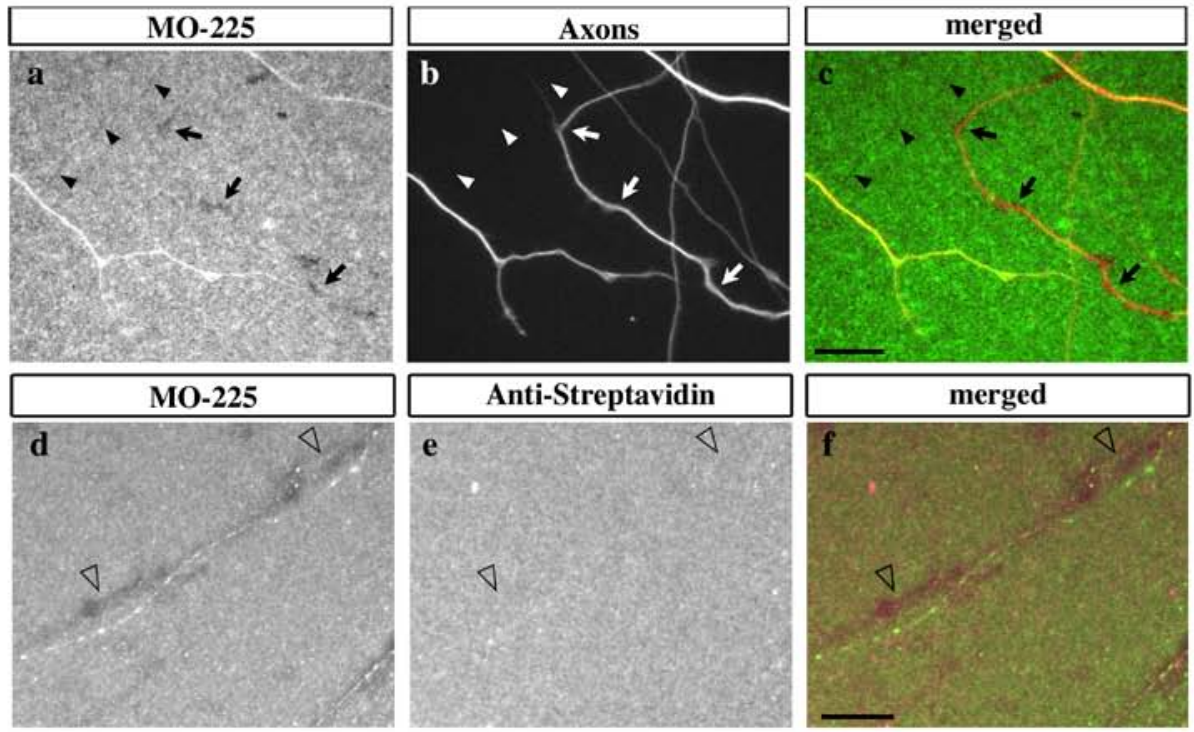

Figure 2 